NUC-MINN-03/4-T

\title{
Rate Equation Network for Baryon Production in High Energy Nuclear Collisions
}

\author{
Pasi Huovinen ${ }^{1,2,3}$ and Joseph Kapusta ${ }^{1}$ \\ ${ }^{1}$ School of Physics and Astronomy, University of Minnesota \\ Minneapolis, Minnesota 55455, USA \\ ${ }^{2}$ Helsinki Institute of Physics, P.O. Box 64 \\ FIN-00014 University of Helsinki, Finland \\ ${ }^{3}$ Department of Physics, P.O. Box 35 \\ FIN-40014 University of Jyväskylä, Finland
}

\begin{abstract}
We develop and solve a network of rate equations for the production of baryons and anti-baryons in high energy nuclear collisions. We include all members of the baryon octet and decuplet and allow for transformations among them. This network is solved during a relativistic $2+1$ hydrodynamical expansion of the of the hot matter created in the collision. As an application we compare to the number of protons, lambdas, negative cascades, and omega baryons measured at mid-rapidity in central collisions of gold nuclei at $65 \mathrm{GeV}$ per nucleon at the Relativistic Heavy Ion Collider (RHIC).
\end{abstract}

PACS: 12.38.Mh, 25.75.Dw, 25.75.-q 


\section{INTRODUCTION}

It has been a challenge to understand the relatively high abundances of baryons and antibaryons produced in high energy gold-gold collisions at the Brookhaven National Laboratory RHIC (Relativistic Heavy Ion Collider). The center of mass collision energies have ranged between 56 and $200 \mathrm{GeV}$ per nucleon pair. This energy is so high that baryon/anti-baryon pairs can readily be created. To very good accuracy the relative abundances, including multiply strange hyperons, are consistent with them being in chemical equilibrium at a temperature of $170 \pm 10 \mathrm{MeV}$ and a baryon chemical potential on the order of tens of $\mathrm{MeV}[1,2]$. Furthermore, the ratio of baryons to mesons grows with increasing transverse momentum, reaching one at $p_{T} \approx 2 \mathrm{GeV} / \mathrm{c}[3]$. A frequently given explanation of the first result is that the hadrons are created in chemical equilibrium at some temperature and chemical potential, possibly at the end of a quark-gluon to hadron phase transition. In the absence of detailed information on the dynamics it is quite reasonable to postulate that phase space is filled randomly. For a big system, this is equivalent mathematically to momentary or instantaneous thermal and chemical equilibrium. A possible explanation of the second result is that since the transverse momentum is proportional to mass, collective fluid-like flow velocity of the expanding matter would boost heavier particles to higher transverse

momentum. A nice overview of these results can be obtained from the Proceedings of Quark Matter 2002 [4].

The results on baryon production at RHIC are exciting and stimulating. A parametrization of them in terms of temperature $T$, baryon chemical potential $\mu$ arising from the initial baryon number of the gold nuclei, and transverse flow velocity $v_{T}$ is a first step. The next step is to understand them in terms of microscopic, dynamical processes. In this paper we set up a network of rate equations to describe the production of baryon/anti-baryon pairs (such as $p \bar{\Lambda}$ and $\Sigma \bar{\Delta}$ ) including all members of the octet and decuplet. We also include transformations among them (such as $\Delta^{+} \leftrightarrow p \pi^{0}$ ). The rate of production of octet-octet pairs was derived by Kapusta and Shovkovy [5]. To compare with experimental data it is 
important to include the production of octet-decuplet and decuplet-decuplet pairs too. Unfortunately there is insufficient experimental information to determine these as accurately. Therefore, we use a simple form of $\mathrm{SU}(3)$ flavor symmetry to estimate these rates. Then we solve the network of rate equations in a $2+1$ dimensional hydrodynamical model of the expanding high energy density matter in heavy ion collisions which has been tuned to reproduce the measured pion multiplicity [3]. Since this is a set of coupled differential equations initial conditions are required. We begin to solve the rate equations in the mixed phase of a first order quark-gluon to hadron phase transition with three values of $T_{c}$, namely, 165 , 180, and $200 \mathrm{MeV}$. In one scenario we assume that all baryons are in chemical equilibrium initially, and in the other we assume no baryons initially. Other scenarios are certainly possible. We plot the resulting abundances as a function of the local (freeze-out) temperature $T_{f}$ and compare to the measured numbers of $p, \Lambda, \Xi^{-}$, and $\Omega$ in central gold-gold collisions at mid-rapidity at a beam energy of $65 \mathrm{GeV}$ per nucleon at RHIC. We also study what happens when the octet-decuplet and decuplet-decuplet rates are increased or decreased by a factor of two. Comparison to the transverse momentum distributions of these baryons will be done in a later paper.

\section{NETWORK OF RATE EQUATIONS}

We need to specify the dynamical variables, measure or calculate the microscopic rates, and then solve the resulting network of rate equations. We shall do each of these in turn.

Various members of the baryon (or anti-baryon) octet and decuplet have been or will be measured in central heavy ion collisions at the CERN Super Proton Synchrotron (SPS) and at RHIC. Therefore the variables will be all members of those multiplets. There are many mesons that are much lighter than the baryons, such as the pion and the $\rho$ and $\omega$ vector mesons to name just a few. The baryons are also much heavier than the three lightest species of quarks when the latter are given their current quark masses. Therefore it ought to be a good approximation to consider the mesons and/or the quarks and gluons as providing 
a thermal bath in which baryon/anti-baryon pairs can be created or destroyed. Since kinetic equilibrium is usually reached much quicker than chemical equilibrium it is reasonable to take the kinetic energy distribution of the baryons and anti-baryons as approximately thermal. We assume in this paper that only the absolute number of each species deviates from local chemical equilibrium. These approximations can be relaxed but, as they are, the calculations become increasingly complex. Ultimately one would reach the point where microscopic cascade calculations would be necessary, and then one encounters the problem of multiparticle initial states in the quasi-localized interactions. That problem is largely avoided in the present approximation because of the thermal averaging of the microscopic rates (see below).

Under the above conditions, the rate equation for the spatial density of a baryon species $b$ is

$$
\begin{aligned}
\frac{d n_{b}}{d t}= & \sum_{\bar{b}^{\prime}} R\left(b \bar{b}^{\prime}\right)\left[1-\frac{n_{\bar{b}^{\prime}} n_{b}}{n_{\bar{b}^{\prime}}^{\text {equil }} n_{b}^{\text {equil }}}\right]-\frac{n_{b}}{V} \frac{d V}{d t} \\
& +\sum_{b^{\prime}} \Gamma\left(b^{\prime} \rightarrow b+X\right)\left[n_{b^{\prime}}-\frac{n_{b^{\prime}}^{\text {equil }} n_{b}}{n_{b}^{\text {equil }}}\right]-\sum_{b^{\prime}} \Gamma\left(b \rightarrow b^{\prime}+X\right)\left[n_{b}-\frac{n_{b}^{\text {equil }} n_{b^{\prime}}}{n_{b^{\prime}}^{\text {equil }}}\right]
\end{aligned}
$$

The first term on the right involves the rate for producing the specified baryon/anti-baryon pair by strong interaction currents, $R\left(b \bar{b}^{\prime}\right)=$ the number of such pairs produced per unit volume per unit time, and is the driving term in the rate equation. Of course, the same pair can annihilate each other, and this is related to the production rate by detailed balance. The factor in square brackets enforces detailed balance. The $n_{b^{\prime}}^{\text {equil }}$ is the equilibrium density of the species $b^{\prime}$ as represented by the temperature and chemical potentials at the time $t$. The second term is a dilution term; the density will decrease in inverse proportion to the volume for an expanding system. The third term arises if there exists a baryon species that can decay via the strong interactions into the baryon species of interest. The quantity in square brackets following it allows for the inverse reaction and satisfies detailed balance. The last term arises if the baryon species of interest can decay into another baryon via the strong interactions. There is one first order in time, nonlinear, rate equation for each species of 
baryon. This makes a coupled network of differential equations.

The decay rate $\Gamma\left(b^{\prime} \rightarrow b+X\right)$ is just the inverse lifetime for the specified decay, namely, $1 / \tau\left(b^{\prime} \rightarrow b+X\right)$. These are taken from the Particle Data Tables [6]. We include the following decays: $\Delta \rightarrow N+\pi, \Sigma^{*} \rightarrow \Lambda+\pi, \Sigma^{*} \rightarrow \Sigma+\pi, \Xi^{*} \rightarrow \Xi+\pi$.

The rate $R$ is the crucial ingredient in this network of rate equations. It was derived in quite some detail by Kapusta and Shovkovy [5] for the case of baryons and anti-baryons in the lowest octet. The basis for that calculation involved an effective current-current interaction between the baryons and strong interaction currents such as the vector and axial-vector. A combination of hadron phenomenology, experimental data on cross sections, and SU(3) flavor symmetry allows the rates to be expressed in terms of the spectral densities of the strong interaction currents. Since the $\sqrt{s}$ is so large, with a minimum value determined by threshold production of the baryon/anti-baryon pair, these spectral densities can be taken from perturbative QCD. For the production of baryons in the octet, the rate was expressed as $[5]$

$$
R_{8,8}\left(b_{1} \bar{b}_{2}\right)=C_{+}\left(b_{1} \bar{b}_{2}\right) \mathcal{R}_{+}\left(b_{1} \bar{b}_{2}\right)+C_{-}\left(b_{1} \bar{b}_{2}\right) \mathcal{R}_{-}\left(b_{1} \bar{b}_{2}\right)
$$

Here

$$
\begin{aligned}
\mathcal{R}_{ \pm}\left(b_{1} \bar{b}_{2}\right)= & \frac{9\left(1+\alpha_{s} / \pi\right) T^{8}}{4(2 \pi)^{5} f_{\pi}^{4}} z_{1}^{2} z_{2}^{2}\left\{4 z_{1} K_{1}\left(z_{1}\right) K_{2}\left(z_{2}\right)+4 z_{2} K_{1}\left(z_{2}\right) K_{2}\left(z_{1}\right)\right. \\
& \left. \pm\left(z_{1} \pm z_{2}\right)^{2} K_{1}\left(z_{1}\right) K_{1}\left(z_{2}\right)+\left[16+\left(z_{1} \pm z_{2}\right)^{2}\right] K_{2}\left(z_{1}\right) K_{2}\left(z_{2}\right)\right\} F_{\text {ANN }}^{2}(\bar{s})
\end{aligned}
$$

where $z_{i}=m_{i} / T, T$ is the temperature, and $F_{\mathrm{ANN}}^{2}(s)$ is an annihilation form factor evaluated at the average value $\bar{s}=\left(m_{1}+m_{2}\right)^{2}+3\left(m_{1}+m_{2}\right) T$. A simple parametrization of this form factor which is consistent with nucleon/anti-nucleon annihilation data was found to be

$$
F_{\mathrm{ANN}}(s)=\frac{1}{2.21+\left[s-\left(m_{1}+m_{2}\right)^{2}\right] / \Lambda^{2}}
$$

with $\Lambda=1.63 \mathrm{GeV}$. According to the latest data analysis [6] the strong interaction coupling is $\alpha_{s}\left(m_{\tau}^{2}\right)=0.35 \pm 0.03$. The \pm correspond to vector/axial-vector contributions to the rate. The numerical coefficients $C_{ \pm}\left(b_{1} \bar{b}_{2}\right)$ are given in [5]; they are all of order one. 
There is unlikely ever to be sufficient experimental information from hadron-hadron scattering to pin down the production rates for baryons in the decuplet since they are all unstable with lifetimes less than $10^{-10}$ seconds. Therefore, we have estimated the rates and parametrize them as follows.

$$
\begin{aligned}
R_{8,10}\left(b_{1} \bar{b}_{2}\right) & =C_{8,10}\left(b_{1} \bar{b}_{2}\right) \mathcal{R}\left(b_{1} \bar{b}_{2}\right) \\
R_{10,10}\left(b_{1} \bar{b}_{2}\right) & =C_{10,10}\left(b_{1} \bar{b}_{2}\right) \mathcal{R}\left(b_{1} \bar{b}_{2}\right)
\end{aligned}
$$

We take the coefficients of the vector and axial-vector contributions to be equal which means that

$$
\begin{aligned}
\mathcal{R}\left(b_{1} \bar{b}_{2}\right)= & \mathcal{R}_{+}\left(b_{1} \bar{b}_{2}\right)+\mathcal{R}_{-}\left(b_{1} \bar{b}_{2}\right) \\
= & \frac{9\left(1+\alpha_{s} / \pi\right) T^{8}}{2(2 \pi)^{5} f_{\pi}^{4}} z_{1}^{2} z_{2}^{2}\left\{4 z_{1} K_{1}\left(z_{1}\right) K_{2}\left(z_{2}\right)+4 z_{2} K_{1}\left(z_{2}\right) K_{2}\left(z_{1}\right)\right. \\
& \left.+2 z_{1} z_{2} K_{1}\left(z_{1}\right) K_{1}\left(z_{2}\right)+\left[16+z_{1}^{2}+z_{2}^{2}\right] K_{2}\left(z_{1}\right) K_{2}\left(z_{2}\right)\right\} F_{\text {ANN }}^{2}(\bar{s}) .
\end{aligned}
$$

The coefficients $C_{8,10}\left(b_{1} \bar{b}_{2}\right)$ and $C_{10,10}\left(b_{1} \bar{b}_{2}\right)$ are displayed in Tables I and II, respectively. We have constructed them as follows. First, an entry in the table is zero if the quantum numbers of the pair do not match the quantum numbers of the vector or axial-vector meson nonets. Second, exact isospin symmetry within SU(2) multiplets is used. Third, the sum of the coefficients of each column of Table I equals 4, which is the order of magnitude of the corresponding octet-octet coefficients $C_{8,8}$; the sum of the coefficients of each row Table I is either $4 \frac{2}{3}, 5$, or $5 \frac{1}{2}$. Flavor $\mathrm{SU}(3)$ symmetry cannot give more information than this. Similarly the sum of the coefficients of each column (and row) of Table II equals 6 . In our numerical work we shall study the consequences of increasing or decreasing these coefficients overall by a factor of 2 .

There will always be more baryons than anti-baryons present in the final state of a heavy ion collision because the colliding nuclei have a net baryon number. However, this asymmetry is reduced as the beam energy increases because produced baryons always come with an antibaryon due to baryon number conservation. The pair production is an increasing function of beam energy and so the initial number of baryons from the nuclei becomes a smaller and 
smaller fraction of the total. At the highest energies at RHIC the baryon chemical potential is usually estimated to be on the order of $10 \mathrm{MeV}$ when the temperature is $150 \mathrm{MeV}$. For simplicity of calculation and presentation we shall take the baryon chemical potential to be exactly zero in this paper. To remove the difference between the experimental data and our calculation due to this approximation, we compare our results to the observed average yield of a baryon and antibaryon, for example $(p+\bar{p}) / 2$, not to the observed baryon yield as such.

\section{COMPARISON TO DATA}

Baryon and anti-baryon production cannot begin until the local energy density is low enough, that is, when the matter is in the hadronic phase as opposed to the quark-gluon phase. This will occur during the expansion stage of a high energy nucleus-nucleus collision. This stage is frequently modeled with hydrodynamics [7]. We shall do so in this paper too. We will use a $2+1$ dimensional description of the final stage expansion that takes into account transverse expansion. The details of these calculations have been given many times before and so we just refer the reader to those papers [8]. Briefly, the expansion begins in the quark-gluon plasma phase with an energy density adjusted to reproduce the measured pion rapidity density at mid-rapidity. The phase transition is first order; we have chosen $T_{c}$ to be 165,180 , and $200 \mathrm{MeV}$ and test the sensitivity of baryon production to it.

The network of rate equations is solved within each co-moving cell in coordinate space. Different cells evolve somewhat differently in space and time, and this makes for a very computationally intense task. At each point in time, as measured by an observer at rest in the center-of-momentum frame of the central gold-gold collision, each cell has its own temperature. This local temperature enters in the production rates and in the local chemical equilibrium densities. In order to concisely display the results of our calculations we plot the solutions to the network of rate equations as a function of the local temperature and not as a function of the local time. Because each fluid cell has its own temperature at any given time, we must integrate over a constant temperature hypersurface to get the baryon rapidity 
density at this temperature. Thus the value at a fixed temperature does not correspond to any particular time. This choice is rather natural since baryon production begins at a systemwide value of the temperature equal to $T_{c}$ and ends at a supposed freeze-out temperature $T_{f}$ where the hadrons lose local thermal equilibrium and begin their free-streaming stage.

In figure 1 we display the results of numerical solution of the rate equation network for $p$, $\Lambda, \Xi^{-}$and $\Omega$. Plotted is the ratio of the calculated density to the equilibrium density at that particular temperature. No weak decays of unstable baryons have been allowed for at this point. The rate equations require an initial condition and we have chosen two: all baryons in chemical equilibrium at $T_{c}$, and no baryons present at all at $T_{c}$. The former are represented in the figure by the upper set of thick curves while the latter is represented by the lower set of thin curves. Obviously this calculation has used $T_{c}=180 \mathrm{MeV}$, but it is representative of other choices. When baryons are present initially with their equilibrium abundances, they evolve in such a way that they always stay above the equilibrium abundance at each temperature below $T_{c}$. The reason is that the system expands more rapidly than chemical reactions can keep up with. In other words, the typical annihilation rates are smaller than the expansion rate. When no baryons are present initially, their abundance at first builds up rapidly. The reason that there is a finite abundance at $T_{c}$ already is that the cells generally remain within the mixed phase at $T_{c}$ for a finite time span, thus allowing a buildup before the temperature drops below $T_{c}$. In the range from 120 to $130 \mathrm{MeV}$, these baryon species have caught up to the equilibrium abundance at that temperature. Thereafter they are above the equilibrium values for the same reason as stated above, namely, that annihilation rates are generally smaller than the expansion rate. Naturally, the abundances with no baryons present initially never catch up with the abundances where baryons were initially created in chemical equilibrium.

In figures 2-5 we show the rapidity density at mid-rapidity as a function of freeze-out temperature for $p, \Lambda, \Xi^{-}$and $\Omega$, respectively. The upper set of curves result from having baryons in chemical equilibrium initially, while the lower set of curves result from having no baryons present initially. The central curve in each set results from using the standard set of 
octet-decuplet and decuplet-decuplet coefficients shown in the Tables. The upper and lower curves in the lower set result from increasing and decreasing these coefficients by a factor of 2 respectively (the octet-octet coefficients are left unchanged). In the upper set of curves the effect is reversed: the larger rates lead to smaller yields. Also shown in these figures are the experimental data from RHIC experiments. Because we use the approximation of zero baryon chemical potential, we compare our result to the measured average yield of baryon and antibaryon. The $(p+\bar{p}) / 2$ and $(\Lambda+\bar{\Lambda}) / 2$ data come from PHENIX [9] and the $\left(\Xi^{-}+\bar{\Xi}^{+}\right) / 2$ and $(\Omega+\bar{\Omega}) / 2$ data come from STAR [10]. The calculation and the PHENIX data are for $5 \%$ most central collisions whereas the STAR data are for $10 \%$ most central collisions. By comparing the hydrodynamically calculated pion yields at different centralities we have estimated that this leads to about $10 \%$ larger yield in our calculation compared to the data. The darker central band in each figure represents statistical errors only while the lighter outer band includes systematic errors as well. (For $\Omega$ the systematic error is smaller than the statistical error.) We have performed decays of unstable baryons appropriate to the particular measurement. For example, protons coming from the weak decay $\Lambda \rightarrow p+\pi^{-}$ are not included in figure 2 , but protons from the decay $\Sigma^{+} \rightarrow p+\pi^{0}$ are.

What we learn from this set of figures is that the baryon production rates are too small in comparison to the expansion rate to reproduce any of the experimental data when there are no baryons present initially. There must be a significant abundance of baryons present already at the beginning of the hadronic phase. Assuming the baryons to be produced in chemical equilibrium and then evolving them during the expansion of the matter provides agreement with the data on $p, \Lambda$, and $\Xi^{-}$within systematic error bars for $120 \leq T_{f} \leq$ $T_{c}$. However, the theoretical calculations produce about $50 \%$ more $\Omega$ than observed. It is interesting that this magnitude of discrepancy does not occur for the $\Xi^{-}$, even though it has two valence s-quarks. Oftentimes in purely statistical models an s-quark fugacity factor is used to fit the data. A typical value might be 0.9 , so that the $\Lambda$ yield would be multiplied by 0.9 , the $\Xi^{-}$yield by $(0.9)^{2}$, and the $\Omega$ yield by $(0.9)^{3}$. Any such suppression factor should be an outcome of the results of a rate equation calculation like the present one, and cannot 
be done arbitrarily at the end of the calculation to fit data. On the other hand, if one had confidence that there was a first-order phase transition with this numerical value of $T_{c}$ and that the expansion was described adequately by hydrodynamics, then one could adjust the initial conditions to match the experimental data.

Next we test the sensitivity of the results to the critical temperature. The results are shown in figures 6-9. In these calculations we have used the set of coefficients from Tables I and II. The calculated abundances are generally increasing functions of $T_{c}$, chosen here to be 165, 180 and $200 \mathrm{MeV}$. As seen in figures 2-5, the abundances calculated when no baryons are present initially are all below the data. When the baryons are in chemical equilibrium initially, the data on $\Lambda$ and $\Xi^{-}$are bracketed by the curves. The $p$ are consistent, within systematic errors, with 180 and $200 \mathrm{MeV}$. The $\Omega$ data is consistent with $165 \mathrm{MeV}$ but is below the 180 and $200 \mathrm{MeV}$ curves. However, there is no value of $T_{c}$ which provides agreement between the calculated abundance and the observed one for all four species of baryons.

\section{CONCLUSION}

In this paper we have estimated the thermal production rates of baryon/anti-baryon pairs when one or both of them are members of the spin-3/2 decuplet. This estimate is based on the thermal production rates when both are members of the spin-1/2 octet [5]. The latter rates are quite well-founded in hadronic phenomenology and QCD, whereas the rates involving the spin-3/2 baryons are somewhat uncertain as they are unstable and beams cannot be made to measure cross sections. We used exact isospin symmetry within multiplets; flavor SU(3) was used too, but it is insufficient to pin down all the rate coefficients. We made numerical estimates based on octet-octet production and ultimately tested the sensitivity of the results to changes of a factor 2 in the coefficients.

We used 2+1 dimensional hydrodynamics, which includes transverse expansion, to model the expansion stage of a central gold-gold collision at RHIC. This provided the thermal bath 
within which the network of rate equations was solved. The network of equations requires initial conditions. We chose two interesting limits to study in detail: no baryons present initially and baryons initially in chemical equilibrium. We also studied the sensitivity of the results to the critical temperature of an assumed first order phase transition from quarks and gluons to hadrons.

When comparison is made to RHIC data on $p, \Lambda, \Xi^{-}$and $\Omega$, the most important conclusion is that the calculated production rates are too small to compensate for the rapid expansion of the matter as described by hydrodynamics if there are no baryons present at the end of the phase transition. There is just not enough time to build up the abundances of these baryons starting from nothing. On the other hand, the observed abundances of $p$, $\Lambda$, and $\Xi^{-}$can be reproduced when this network of rate equations is solved within a hydrodynamic expansion if baryons are taken to be in chemical equilibrium when each fluid cell is converted from quarks and gluons to hadrons. The observed abundance of $\Omega$, however, is generally less than the calculated one, and when the parameters are chosen to obtain the correct $\Omega$ abundance the other baryons are underproduced. So the $\Omega$ remains a bit of a puzzle.

The results obtained here are not trivial nor are they obvious. The interplay among the initial conditions, the expansion dynamics (in particular the expansion rate), and the production rates of octet and decuplet baryons is intricate and subtle. If the baryons are produced in chemical equilibrium at $T_{c}$, their numbers will decrease as the system expands and cools by an amount that depends on the annihilation rates compared to the expansion rate. If no baryons are present at $T_{c}$, their numbers will at first increase with time until local equilibrium is achieved at some temperature below $T_{c}$ which is generally different for each species. After that there will be an overabundance of baryons relative to equilibrium, and their numbers will begin to decrease.

Among the extensions of this project under investigation are different choices of the initial abundances and the transverse momentum distributions of the various baryon species. The latter would allow us to study the expansion dynamics in finer details than just the overall 
$\mathrm{dN} /$ dy at mid-rapidity.

\section{ACKNOWLEDGEMENTS}

This work was supported by the US Department of Energy under grant DE-FG0287ER40328. 


\section{REFERENCES}

[1] P. Braun-Munzinger, D. Magestro, K. Redlich, and J. Stachel Phys. Lett. B 518, 41 (2001).

[2] J. Rafelski, J. Letessier, and G. Torrieri, Phys. Rev. C 64, 054907 (2001); Erratum-ibid. 65, $069902(2002)$.

[3] K. Adcox et al. [PHENIX Collaboration], Phys. Rev. Lett. 88, 242301 (2002).

[4] Proceedings of Quark Matter 2002, Nantes, France, July 2002, Nucl. Phys. A 715 (2003).

[5] J. Kapusta and I. Shovkovy, Phys. Rev. C 68, 014901 (2003).

[6] Particle Data Group, D. E. Groom et al., Eur. Phys. J. C 15, 1 (2000).

[7] P. Huovinen, arXiv:nucl-th/0305064; P. F. Kolb and U. Heinz, arXiv:nucl-th/0305084.

[8] P. F. Kolb, P. Huovinen, U. W. Heinz and H. Heiselberg, Phys. Lett. B 500, 232 (2001);

P. F. Kolb, J. Sollfrank and U. W. Heinz, Phys. Rev. C 62, 054909 (2000).

[9] K. Adcox et al. [PHENIX Collaboration], Phys. Rev. Lett. 89, 092302 (2002).

[10] J. Adams et al. [STAR Collaboration], arXiv:nucl-ex/0307024. 
Table I: Relative strengths of coefficients in the expressions for the rates.

\begin{tabular}{|c||c|c|c|c|c|c|c|c|c|c|}
\hline & $\Delta^{++}$ & $\Delta^{+}$ & $\Delta^{0}$ & $\Delta^{-}$ & $\Sigma^{*+}$ & $\Sigma^{* 0}$ & $\Sigma^{*-}$ & $\Xi^{* 0}$ & $\Xi^{*-}$ & $\Omega$ \\
\hline \hline $\bar{p}$ & 2 & $4 / 3$ & $2 / 3$ & 0 & 1 & $1 / 2$ & 0 & 0 & 0 & 0 \\
\hline $\bar{n}$ & 0 & $2 / 3$ & $4 / 3$ & 2 & 0 & $1 / 2$ & 1 & 0 & 0 & 0 \\
\hline $\bar{\Lambda}$ & 0 & 0 & 0 & 0 & 1 & 1 & 1 & 1 & 1 & 0 \\
\hline $\bar{\Sigma}^{+}$ & 2 & $2 / 3$ & 0 & 0 & $1 / 2$ & $1 / 2$ & 0 & 1 & 0 & 0 \\
\hline $\bar{\Sigma}^{0}$ & 0 & $4 / 3$ & $4 / 3$ & 0 & $1 / 2$ & 0 & $1 / 2$ & $1 / 2$ & $1 / 2$ & 0 \\
\hline $\bar{\Sigma}^{-}$ & 0 & 0 & $2 / 3$ & 2 & 0 & $1 / 2$ & $1 / 2$ & 0 & 1 & 0 \\
\hline $\bar{\Xi}^{0}$ & 0 & 0 & 0 & 0 & 1 & $1 / 2$ & 0 & 1 & $1 / 2$ & 2 \\
\hline $\bar{\Xi}^{-}$ & 0 & 0 & 0 & 0 & 0 & $1 / 2$ & 1 & $1 / 2$ & 1 & 2 \\
\hline
\end{tabular}


Table II: Relative strengths of coefficients in the expressions for the rates.

\begin{tabular}{|c||c|c|c|c|c|c|c|c|c|c|}
\hline & $\Delta^{++}$ & $\Delta^{+}$ & $\Delta^{0}$ & $\Delta^{-}$ & $\Sigma^{*+}$ & $\Sigma^{* 0}$ & $\Sigma^{*-}$ & $\Xi^{* 0}$ & $\Xi^{*-}$ & $\Omega$ \\
\hline \hline $\bar{\Delta}^{++}$ & $12 / 5$ & $8 / 5$ & 0 & 0 & 2 & 0 & 0 & 0 & 0 & 0 \\
\hline $\bar{\Delta}^{+}$ & $8 / 5$ & $4 / 15$ & $32 / 15$ & 0 & $2 / 3$ & $4 / 3$ & 0 & 0 & 0 & 0 \\
\hline $\bar{\Delta}^{0}$ & 0 & $32 / 15$ & $4 / 15$ & $8 / 5$ & 0 & $4 / 3$ & $2 / 3$ & 0 & 0 & 0 \\
\hline $\bar{\Delta}^{-}$ & 0 & 0 & $8 / 5$ & $12 / 5$ & 0 & 0 & 2 & 0 & 0 & 0 \\
\hline $\bar{\Sigma}^{*+}$ & 2 & $2 / 3$ & 0 & 0 & $2 / 3$ & $2 / 3$ & 0 & 2 & 0 & 0 \\
\hline $\bar{\Sigma}^{* 0}$ & 0 & $4 / 3$ & $4 / 3$ & 0 & $2 / 3$ & 0 & $2 / 3$ & 1 & 1 & 0 \\
\hline $\bar{\Sigma}^{*-}$ & 0 & 0 & $2 / 3$ & 2 & 0 & $2 / 3$ & $2 / 3$ & 0 & 2 & 0 \\
\hline $\bar{\Xi}^{* 0}$ & 0 & 0 & 0 & 0 & 2 & 1 & 0 & $2 / 3$ & $1 / 3$ & 2 \\
\hline $\bar{\Xi}^{*-}$ & 0 & 0 & 0 & 0 & 0 & 1 & 2 & $1 / 3$ & $2 / 3$ & 2 \\
\hline $\bar{\Omega}$ & 0 & 0 & 0 & 0 & 0 & 0 & 0 & 2 & 2 & 2 \\
\hline
\end{tabular}




\section{FIGURES}

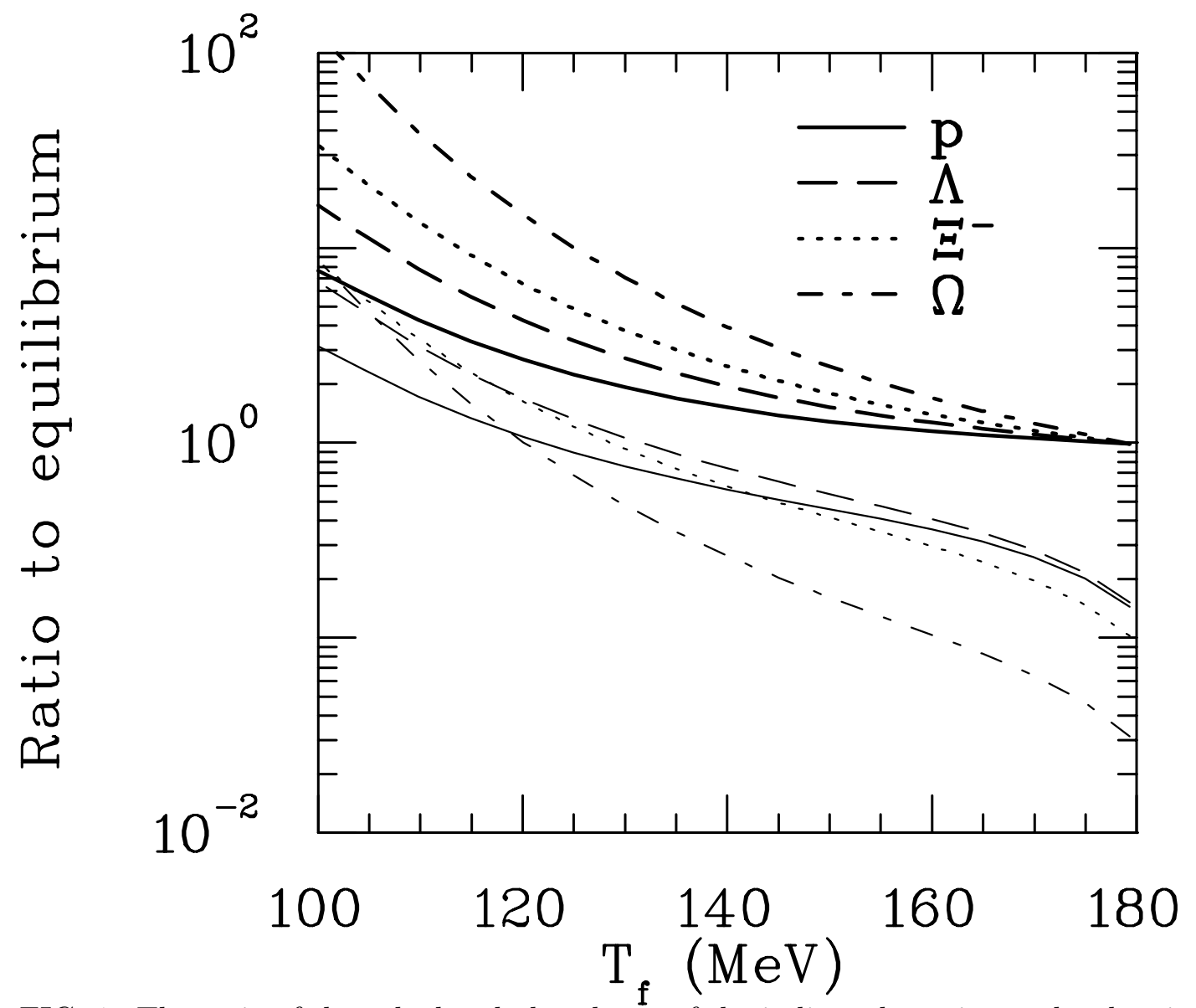

FIG. 1. The ratio of the calculated abundance of the indicated species to the chemical equilibrium value as a function of the local temperature. The upper set of curves start with the baryons in equilibrium at $T_{c}$, the lower set start with no baryons. 


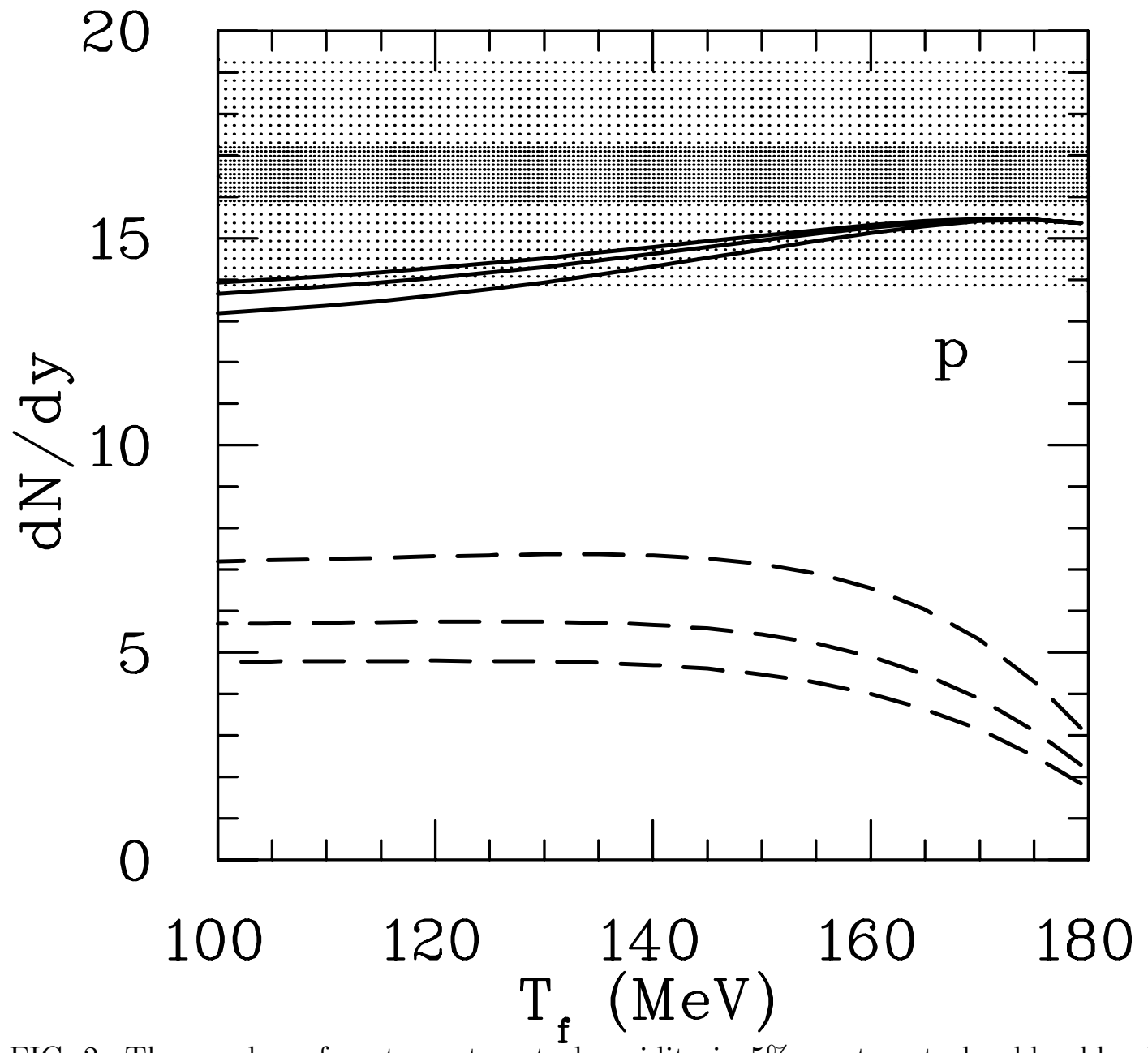

FIG. 2. The number of protons at central rapidity in $5 \%$ most central gold-gold collisions as a function of local temperature. The solid curves start with baryons in equilibrium, the lower set with no baryons. The central curve in each set uses the coupling coefficients given in the Tables, while the other two use values larger and smaller by a factor of two. With the exception of $\Lambda$, baryons unstable to strong or weak decays have been decayed. The data are from the PHENIX collaboration [9] for the same centrality. The dark band represents statistical and the light band systematic errors. 


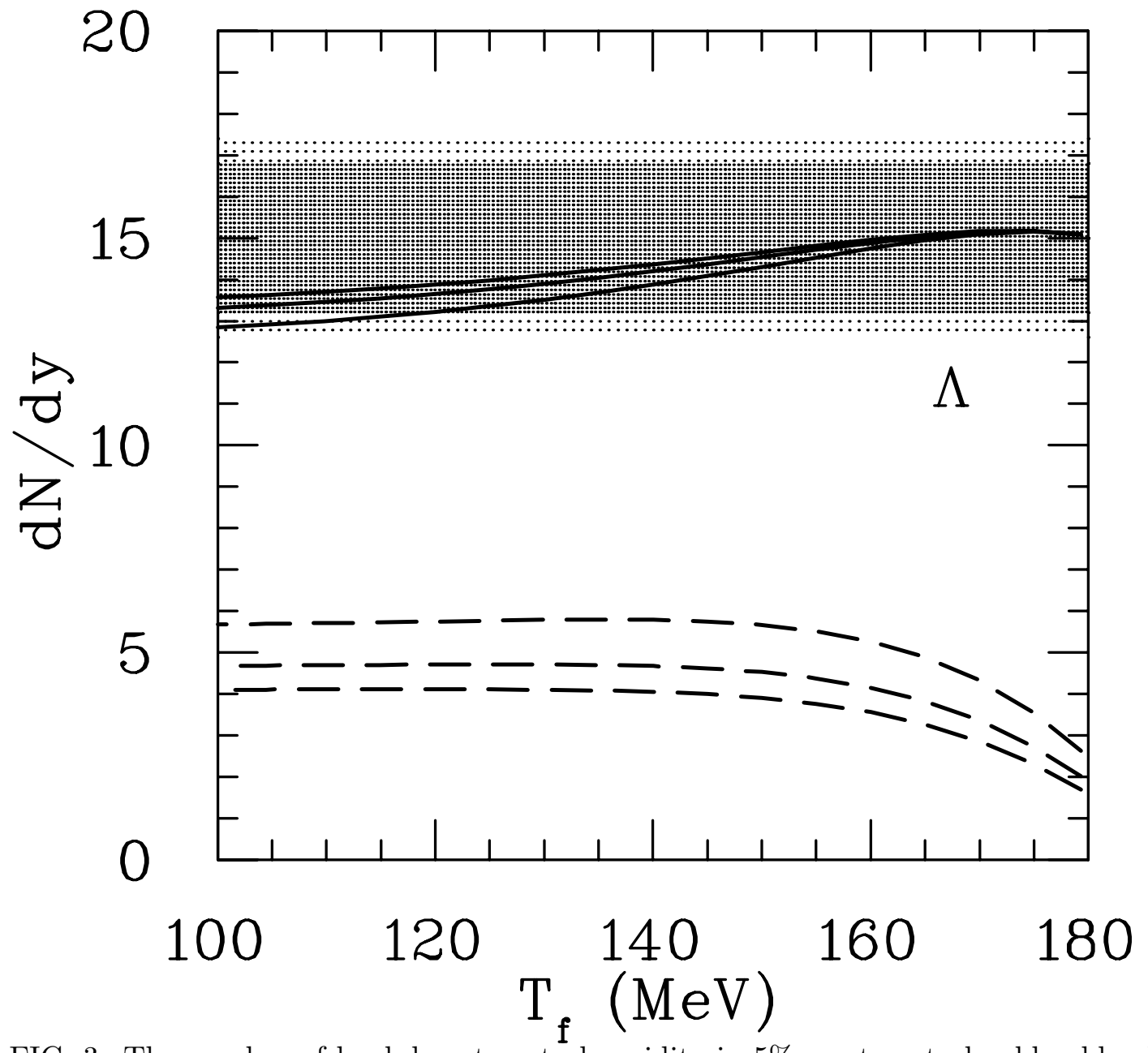

FIG. 3. The number of lambdas at central rapidity in $5 \%$ most central gold-gold collisions as a function of local temperature. The solid curves start with baryons in equilibrium, the lower set with no baryons. The central curve in each set uses the coupling coefficients given in the Tables, while the other two use values larger and smaller by a factor of two. With the exception of $\Lambda$, baryons unstable to strong or weak decays have been decayed. The data are from the PHENIX collaboration [9] for the same centrality. The dark band represents statistical and the light band systematic errors. 


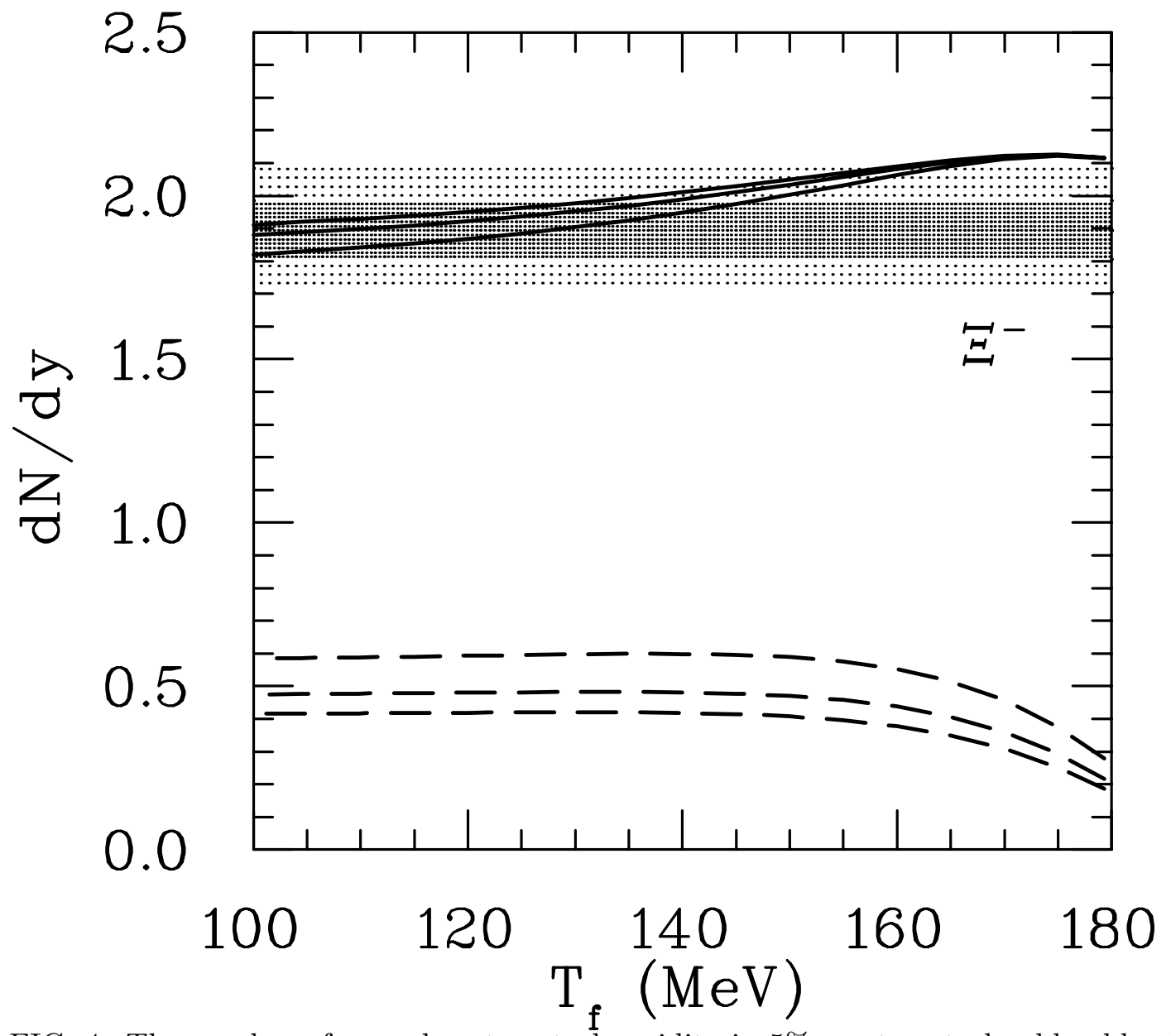

FIG. 4. The number of cascades at central rapidity in 5\% most central gold-gold collisions as a function of local temperature. The solid curves start with baryons in equilibrium, the lower set with no baryons. The central curve in each set uses the coupling coefficients given in the Tables, while the other two use values larger and smaller by a factor of two. Baryons unstable to strong decays have been decayed. The data are from the STAR collaboration [10] for $10 \%$ most central collisions. The dark band represents statistical and the light band systematic errors. 


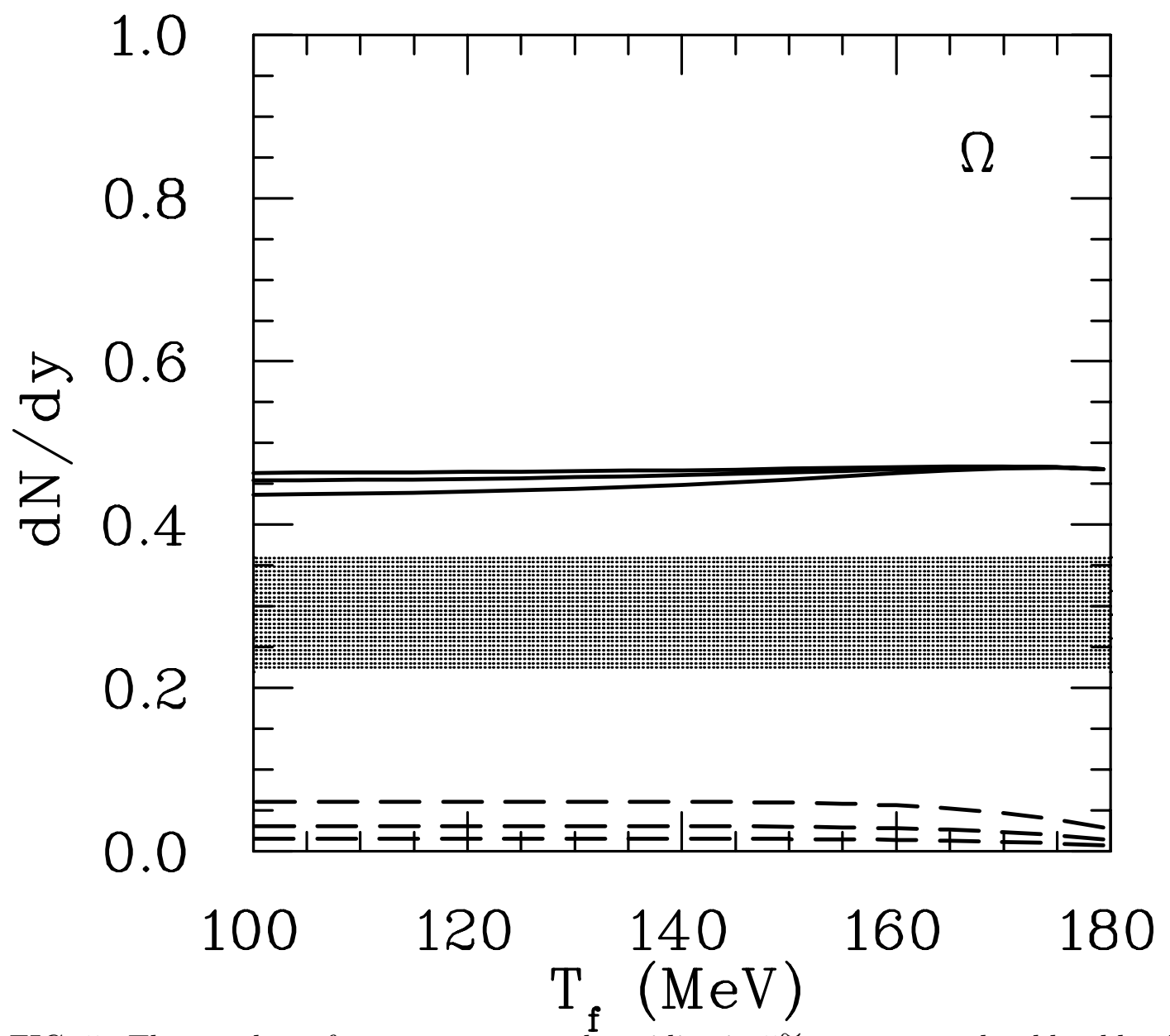

FIG. 5. The number of omegas at central rapidity in 5\% most central gold-gold collisions as a function of local temperature. The solid curves start with baryons in equilibrium, the lower set with no baryons. The central curve in each set uses the coupling coefficients given in the Tables, while the other two use values larger and smaller by a factor of two. Baryons unstable to strong decays have been decayed. The data are from the STAR collaboration [10] for $10 \%$ most central collisions. The dark band represents statistical errors only; the systematic errors are smaller than the statistical ones. 


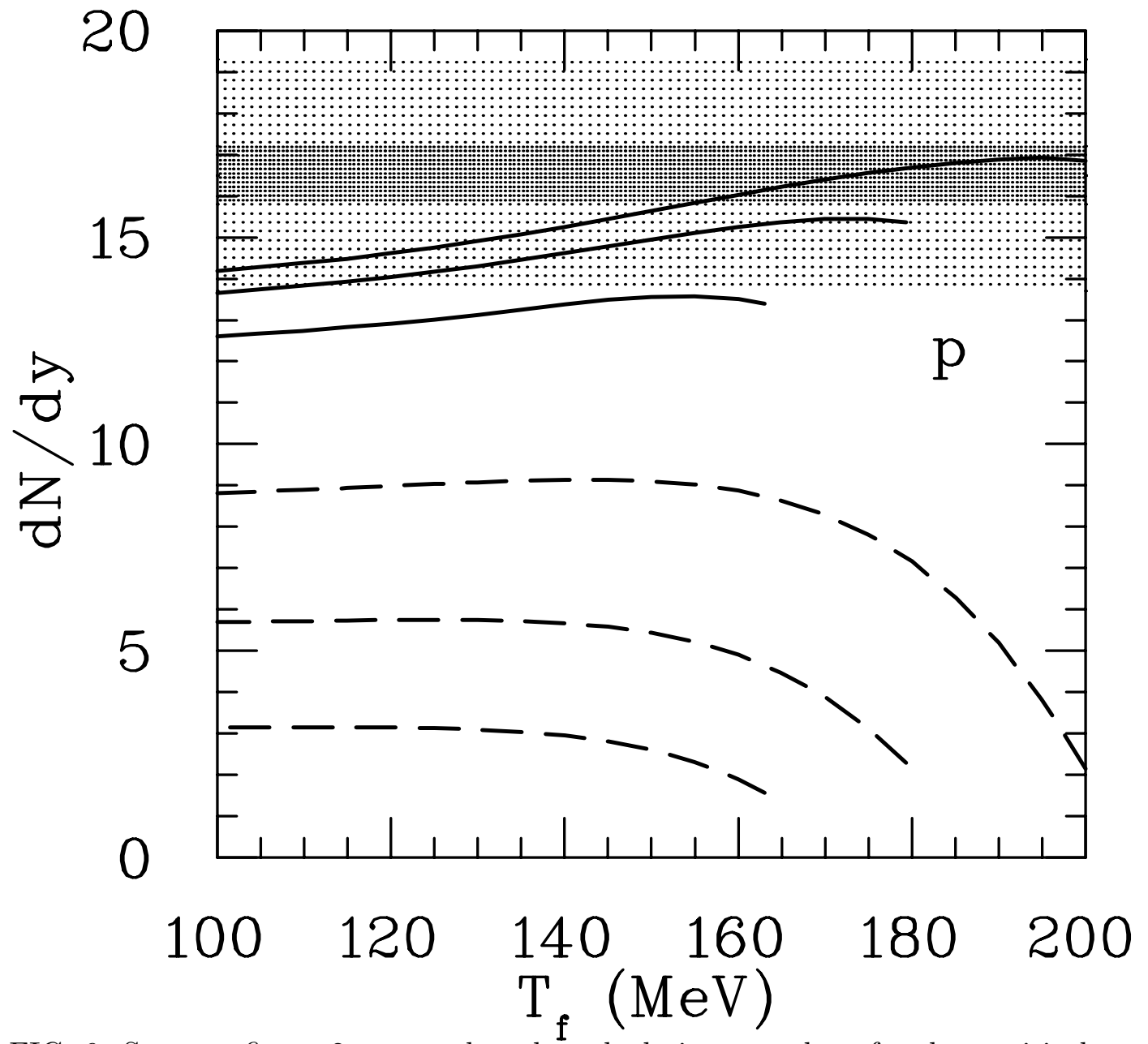

FIG. 6. Same as figure 2 except that the calculations are done for three critical temperatures of 165, 180, and $200 \mathrm{MeV}$. The coefficients from the Tables are used. 


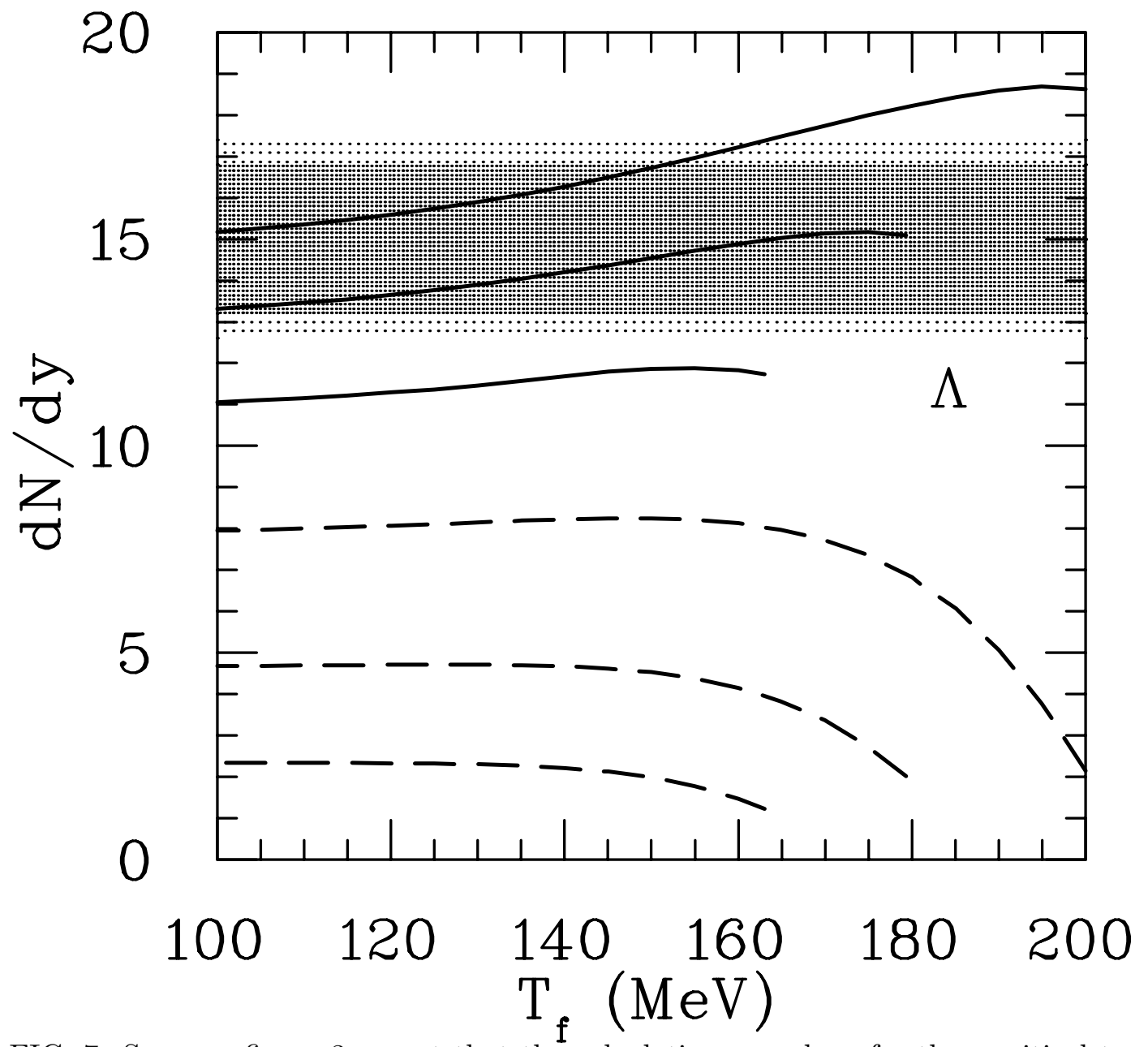

FIG. 7. Same as figure 3 except that the calculations are done for three critical temperatures of 165, 180, and $200 \mathrm{MeV}$. The coefficients from the Tables are used. 


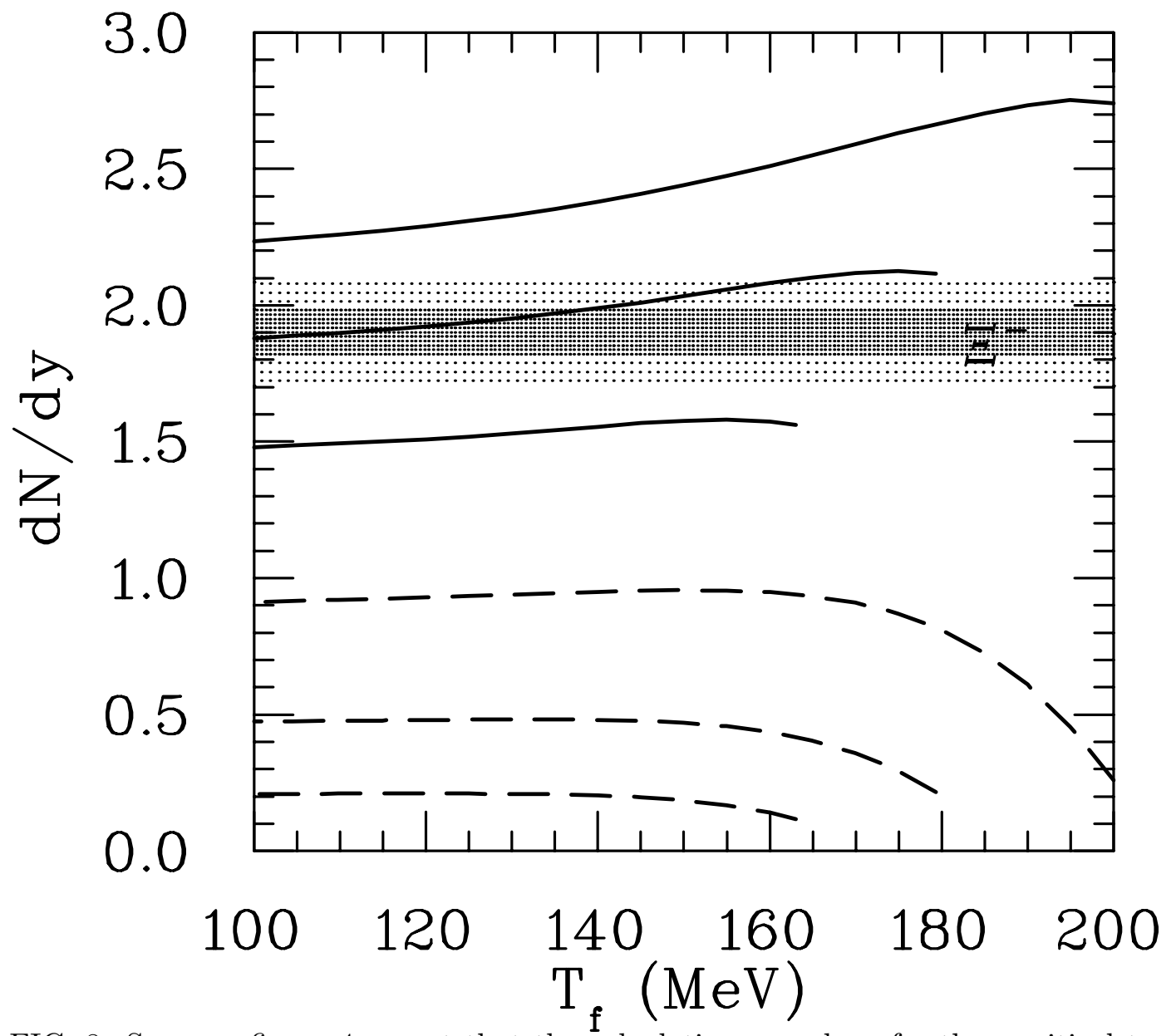

FIG. 8. Same as figure 4 except that the calculations are done for three critical temperatures of 165, 180, and $200 \mathrm{MeV}$. The coefficients from the Tables are used. 


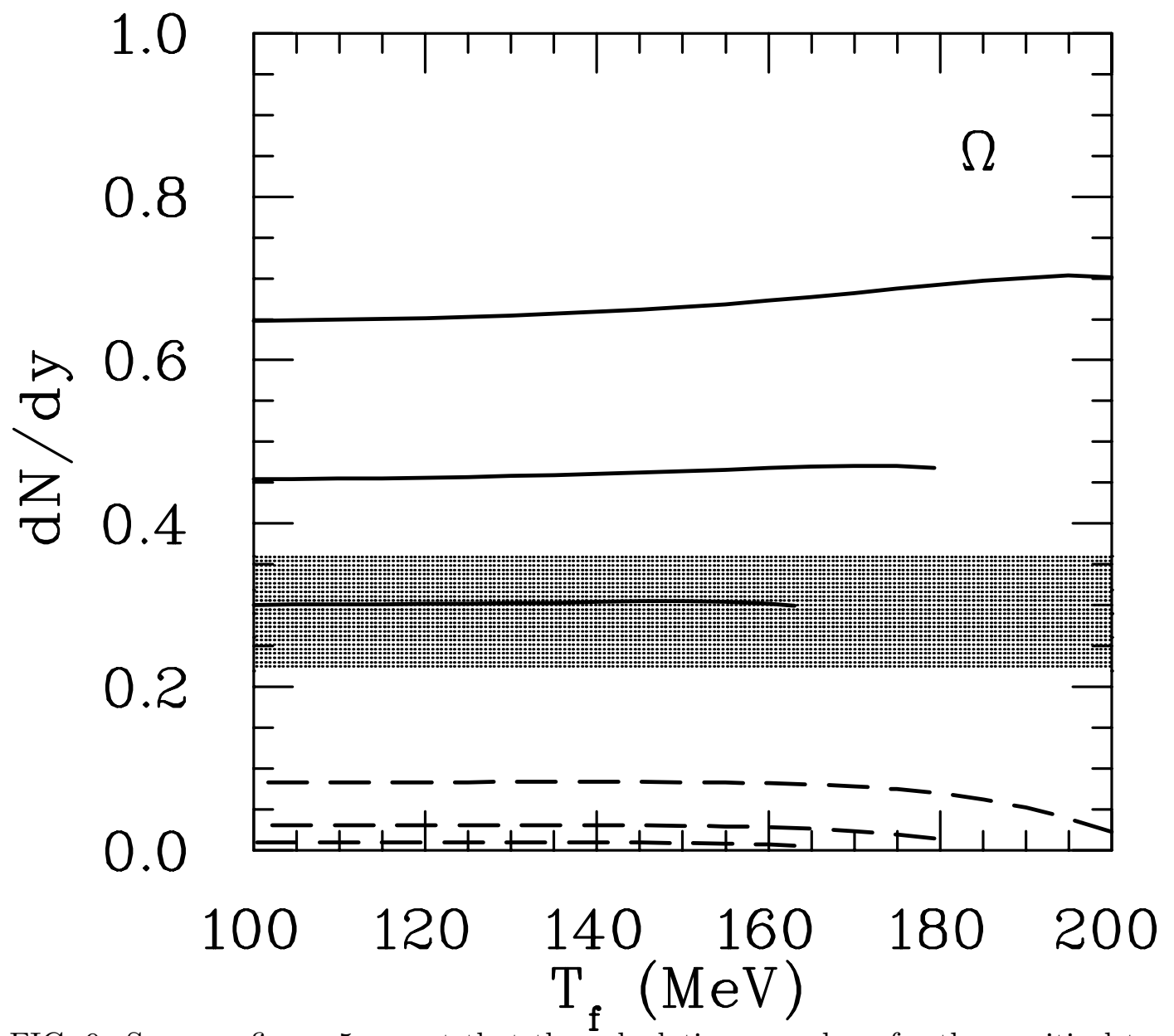

FIG. 9. Same as figure 5 except that the calculations are done for three critical temperatures of 165, 180, and $200 \mathrm{MeV}$. The coefficients from the Tables are used. 